focal generation of seizures but not seizure spread. No change was noted in spatial learning and exploratory behavior tasks. (Hori A, Tandon P, Holmes GL, Stafstrom CE. Ketogenic diet: Effects on expression of kindled seizures and behavior in adult rats. Epilepsia July 1997;38:750-758). (Reprints: Dr CE Stafstrom, Division of Pediatric Neurology, \#330, New England Medical Center, 750 Washington St, Boston, MA 02111).

COMMENT. The renewed interest in the clinical use of the ketogenic diet in the management of childhood epilepsy has stimulated further research in animal models. Some earlier studies of the effect of the KD on experimental seizures and seizure thresholds in laboratory animals showed that seizure susceptibility was not modified in normal animals, but an anticonvulsant effect of the KD was demonstrated in mice with seizure thresholds lowered by water intoxication and hypoelectrolytemia. (Millichap JG, Jones JD, Rudis BP. Mechanism of anticonvulsant action of the ketogenic diet. Amer I Dis Child 1962;104:506, and 1964;107:593-604). In animals and in children with absence epilepsy, the anticonvulsant effect of the KD was unrelated to diuresis, independent of acidosis and ketosis, similar to the effects of acetazolamide, and correlated most closely with a negative balance of sodium and potassium. Additional animal models were reported by Uhlemann ER, Neims AH, 1972; Appleton DB, DeVivo DC, 1974; and Nakazawa M et al, 1983.

Nordli DR Jr, and De Vivo DC provide an editorial commentary. (The ketogenic diet revisited: back to the future. Epilepsia July 1997;38:743-749). Further projected objectives of clinical trials and experimental models are discussed. We know that the ketogenic diet works, but how can we offer the diet or its specific ingredient in a more practical form? Clinical metabolic and laboratory studies should help to achieve this goal.

\title{
NEUROPATHIES
}

\section{PERIPHERAL NEUROPATHY AND HIV INFECTION}

The prevalence and patterns of peripheral neuropathy in 50 children with HIV infection were analyzed retrospectively in the EMG Section, National Institutes of Health, Bethesda, MD. Sensory complaints were the most common reason for referral for EMG. Of 19 children with neurological abnormalities, sensory loss or hypoactive reflexes, 10 had abnormal nerve conduction studies (NCS). In 7 children, NCSs were compatible with a distal symmetric sensory or sensorimotor axonal polyneuropathy (DSPN). All had advanced disease and had received antiretroviral agents; in one the neuropathy was linked to vincristine treatment. Three children had median nerve compression at the carpal tunnel; two had received AZT. Of 2 additional patients with abnormal NCS, one had a demyelinating neuropathy which improved after AZT, and one had a lumbosacral polyradiculopathy associated with varicella zoster involving legs and buttocks. Distal symmetric polyneuropathy affected older children. (Floeter MK, Civitello LA, Everett CR, Dambrosia J, Luciano CA. Peripheral neuropathy in children with HIV infection. Neurology July 1997;49:207-212). (Reprints: Dr MK Floeter, EMG Laboratory, NINDS, NIH, BId 10 RM 5C101, 10 Center Drive MSC 1404, Bethesda, MD 20892).

COMMENT. The various patterns of peripheral neuropathy encountered in children with HIV resemble those described in adults. The most common type is a demyelinating sensory or sensorimotor polyneuropathy. 